\title{
Introduction to the Theory of Computation
}

\author{
Pierre Wolper \\ Email: Pierre.Wolper@ulg.ac.be \\ URL: http: //www.montefiore.ulg.ac.be/ pw/ \\ http: //www.montefiore.ulg.ac.be/ \\ $\sim$ pw/cours/calc.html
}

\section{References}

Pierre Wolper, Introduction à la calculabilité - 3ième édition, Dunod, 2006.

Michael Sipser, Introduction to the Theory of Computation, Second Edition, Course Technology, 2005 
Chapter 1

\section{Introduction}




\subsection{Motivation}

- To understand the limits of computer science.

- To distinguish problems that are solvable by algorithms from those that are not.

- To obtain results that are independent of the technology used to build computers. 


\subsection{Problems and Languages}

- Which problems can be solved by a program executed by a computer?

We need to be more precise about:

- the concept of problem,

- the concept of program executed by a computer. 


\section{The concept of problem}

Problem: generic question.

\section{Examples :}

- to sort an array of numbers;

- to decide if a program written in $\mathrm{C}$ stops for all possible input values; (halting problem);

- to decide if an equation with integer coefficients has integer solutions (Hilbert's 10th problem). 


\section{The concept of program}

Effective procedure: program that can be executed by a computer.

Examples :

- Effective procedure : program written in JAVA ;

- Not an effective procedure: "to solve the halting problem, one must just check that the program has no infinite loops or recursive call sequences." 


\section{The halting problem}

recursive function threen ( $n$ : integer):integer;

begin

if $(n=1)$ then 1

else if even $(n)$ then threen $(n \div 2)$

else threen $(3 \times n+1)$;

end; 


\subsection{Formalizing problems}

How could one represent problem instances? 


\section{Alphabets and words}

Alphabet : finite set of symbols.

\section{Examples}

- $\{\mathrm{a}, \mathrm{b}, \mathrm{c}\}$

- $\{\alpha, \beta, \gamma\}$

- $\{1,2,3\}$

- $\{\boldsymbol{\alpha}, \diamond, \odot\}$ 
Word on an alphabet: finite sequence of elements of the alphabet.

\section{Examples}

- $a, a b s, z t, b b b s s n b n z z y y y y d d t r r a$, grosseguindaille are words on the alphabet $\{a, \ldots, z\}$.

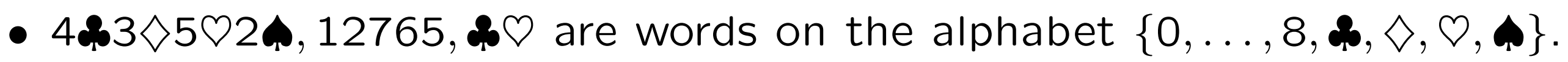

Empty word: represented by $e, \varepsilon$, or $\lambda$.

Length of a word $w:|w|$

$w=a a a b b a a a a b b$

$w(1)=a, w(2)=a, \ldots, w(11)=b$ 


\section{Representing problems}

\section{Encoding a problem}

Let us consider a binary problem whose instances are encoded by words defined over an alphabet $\Sigma$. The set of all words defined on $\Sigma$ can be partitioned in 3 subsets:

- positive instances: the answer is yes ;

- negative instances: the answer is no;

- words that do not represent an instance of the problem. 
Alternatively:

- the words encoding instances of the problem for which the answer is yes, the positive instances;

- the words that do not encode and instance of the problem, or that encode an instance for which the answer is no, the negative instances. 


\section{Languages}

Language: set of words defined over the same alphabet.

\section{Examples}

- $\{a a b, a a a a, \varepsilon, a, b, a b a b a b a b a b b b b b b b b b b b b\},\{\varepsilon, a a a a a a a, a, b b b b b b\}$ and $\emptyset$ (the empty set) are languages over the alphabet $\{a, b\}$.

- for the alphabet $\{0,1\}$,

$\{0,1,00,01,10,11,000,001,010,011,100$,

$101,110,111, \ldots\}$ is the language containing all words.

- language $\emptyset \neq$ language $\{\varepsilon\}$.

- the set of words encoding $C$ programs that always stop. 


\subsection{Describing languages}

\section{Operations on languages}

Let $L_{1}$ and $L_{2}$ be languages.

- $L_{1} \cup L_{2}=\left\{w \mid w \in L_{1}\right.$ or $\left.w \in L_{2}\right\}$;

- $L_{1} \cdot L_{2}=\left\{w \mid w=x y, x \in L_{1}\right.$ and $\left.y \in L_{2}\right\}$;

- $L_{1}^{*}=\left\{w \mid \exists k \geq 0\right.$ and $w_{1}, \ldots, w_{k} \in L_{1}$ such that $\left.w=w_{1} w_{2} \ldots w_{k}\right\}$;

- $\overline{L_{1}}=\left\{w \mid w \notin L_{1}\right\}$. 


\section{Regular Languages}

The set $\mathcal{R}$ of regular languages over an alphabet $\Sigma$ is the smallest set of languages such that:

1. $\emptyset \in \mathcal{R}$ and $\{\varepsilon\} \in \mathcal{R}$,

2. $\{a\} \in \mathcal{R}$ for all $a \in \Sigma$, and

3. if $A, B \in \mathcal{R}$, then $A \cup B, A \cdot B$ and $A^{*} \in \mathcal{R}$. 


\section{Regular expressions}

A notation for representing regular languages.

1. $\emptyset, \varepsilon$ and the elements of $\Sigma$ are regular expressions;

2. If $\alpha$ and $\beta$ are regular expressions, then $(\alpha \beta),(\alpha \cup \beta),(\alpha) *$ are regular expressions.

The set of regular expressions is a language over the alphabet $\Sigma^{\prime}=\Sigma \cup\{),(, \emptyset, \cup, *, \varepsilon\}$. 


\section{The language represented by a regular expression}

1. $L(\emptyset)=\emptyset, L(\varepsilon)=\{\varepsilon\}$,

2. $L(a)=\{a\}$ for each $a \in \Sigma$,

3. $L((\alpha \cup \beta))=L(\alpha) \cup L(\beta)$,

4. $L((\alpha \beta))=L(\alpha) \cdot L(\beta)$,

5. $L((\alpha) *)=L(\alpha)^{*}$. 


\section{Theorem}

A language is regular

if and only if

it can be represented by a regular expression. 


\section{Regulars languages : examples}

- The set of all words over $\Sigma=\left\{a_{1}, \ldots, a_{n}\right\}$ is represented by $\left(a_{1} \cup \ldots \cup a_{n}\right)^{*}\left(\right.$ or $\left.\Sigma^{*}\right)$.

- The set of all nonempty words over $\Sigma=\left\{a_{1}, \ldots, a_{n}\right\}$ is represented by $\left(a_{1} \cup \ldots \cup a_{n}\right)\left(a_{1} \cup \ldots \cup a_{n}\right)^{*}$ (or $\Sigma \Sigma^{*}$, or $\left.\Sigma^{+}\right)$.

- the expression $(a \cup b)^{*} a(a \cup b)^{*}$ represents the language containing all words over the alphabet $\{a, b\}$ that contain at least one "a". 


\section{Regulars languages : more examples}

$$
\left(a^{*} b\right)^{*} \cup\left(b^{*} a\right)^{*}=(a \cup b)^{*}
$$

\section{Proof}

- $\left(a^{*} b\right)^{*} \cup\left(b^{*} a\right)^{*} \subset(a \cup b)^{*}$ since $(a \cup b)^{*}$ represents the set of all words built from the characters "a" and "b".

- Let us consider an arbitrary word

$$
w=w_{1} w_{2} \ldots w_{n} \in(a \cup b)^{*} .
$$

One can distinguish 4 cases... 
1. $w=a^{n}$ and thus $w \subset(\varepsilon a)^{*} \subset\left(b^{*} a\right)^{*}$;

2. $w=b^{n}$ and thus $w \subset(\varepsilon b)^{*} \subset\left(a^{*} b\right)^{*}$;

3. $w$ contains both $a$ 's and $b$ 's and ends with $a b$

$$
\begin{aligned}
& \qquad w=\underbrace{a \ldots a b}_{a^{*} b} \underbrace{\ldots b}_{\left(a^{*} b\right)^{*}} \underbrace{a \ldots a b}_{a^{*} b} \underbrace{\ldots b}_{\left(a^{*} b\right)^{*}} \\
& \Rightarrow w \in\left(a^{*} b\right)^{*} \cup\left(b^{*} a\right)^{*} ;
\end{aligned}
$$

4. $w$ contains both $a$ 's and $b$ 's and ends with an $a \Rightarrow$ similar decomposition. 


\subsection{Languages that are not regular}

\section{Fact}

There are not enough regular expressions to represent all languages!

\section{Definition}

Cardinality of a set...

\section{Example}

The sets $\{0,1,2,3\},\{a, b, c, d\},\{\boldsymbol{d}, \diamond, \odot, \boldsymbol{\phi}\}$ all have the same size. There exists a one-one correspondence (bijection) between them, for example $\{(0, \boldsymbol{\alpha}),(1, \diamond),(2, \diamond),(3, \mathbf{p})\}$. 


\section{Denumerable (countably infinite) sets}

\section{Definition}

An infinite set is denumerable if there exists a bijection between this set and the set of natural numbers.

\section{Remark}

Finite sets are all countable in the usual sense, but in mathematics countable is sometimes used to mean precisely countably infinite. 


\section{Denumerable sets: examples}

1. The set of even numbers is denumerable:

$$
\{(0,0),(2,1),(4,2),(6,3), \ldots\} .
$$

2. The set of words over the alphabet $\{a, b\}$ is denumerable: $\{(\varepsilon, 0),(a, 1),(b, 2),(a a, 3),(a b, 4),(b a, 5)$, $(b b, 6),(a a a, 7) \ldots\}$.

3. The set of rational numbers is denumerable: $\{(0 / 1,0),(1 / 1,1),(1 / 2,2),(2 / 1,3),(1 / 3,4)$, $(3 / 1,5), \ldots\}$.

4. The set of regular expressions is denumerable. 


\section{The diagonal argument}

\section{Theorem}

The set of subsets of a denumerable set is not denumerable.

\section{Proof}

\begin{tabular}{l|cccccc} 
& $a_{0}$ & $a_{1}$ & $a_{2}$ & $a_{3}$ & $a_{4}$ & $\cdots$ \\
\hline$s_{0}$ & $\times$ & $\times$ & & $\times$ & & \\
$s_{1}$ & $\times$ & $\square$ & & $\times$ & & \\
$s_{2}$ & & $\times$ & $\times$ & & $\times$ & \\
$s_{3}$ & $\times$ & & $\times$ & $\square$ & & \\
$s_{4}$ & & $\times$ & & $\times$ & $\square$ \\
$\vdots$ & & & & & \\
\multicolumn{5}{c}{$D$} & $=\left\{a_{i} \mid a_{i} \notin s_{i}\right\}$
\end{tabular}




\section{Conclusion}

- The set of languages is not denumerable.

- The set of regular languages is denumerable.

- Thus there are (many) more languages than regular languages 


\subsection{To follow ...}

- The notion of effective procedure (automata).

- Problems that cannot be solved by algorithms.

- Problems that cannot be solved efficiently. 